\title{
LINE-1 hypomethylation is neither present in rectal aberrant crypt foci nor associated with field defect in sporadic colorectal neoplasia
}

\author{
Isabel Quintanilla', Maria Lopez-Cerón', Mireya Jimeno², Miriam Cuatrecasas², Jennifer Muñoz ', Leticia Moreira',
} Sabela Carballal', Maria Liz Leoz ${ }^{1}$, Jordi Camps ${ }^{1}$, Antoni Castells ${ }^{1}$, Maria Pellisé ${ }^{* *}$ and Francesc Balaguer ${ }^{1 *}$

\begin{abstract}
Background: Aberrant crypt foci (ACF) are considered the first identifiable preneoplastic lesion in colorectal cancer (CRC), and have been proposed as a potential biomarker for CRC risk. Global DNA hypomethylation is an early event in colorectal carcinogenesis, and long interspersed nuclear element-1 (LINE-1) methylation status is a well-known surrogate marker for genome-wide DNA methylation levels. Despite the gradual increase in DNA hypomethylation in the adenoma-carcinoma sequence, LINE-1 methylation in ACF has never been studied. Moreover, recent studies have reported a field defect for LINE-1 hypomethylation, suggesting that LINE-1 methylation status in normal mucosa could be used to stratify CRC risk and tailor preventive strategies. Thus, we assessed LINE-1 status by pyrosequencing in rectal ACF and paired normal colorectal mucosa from individuals with sporadic colon cancer (CC) $(n=35)$ or adenoma $(n=42)$, and from healthy controls $(n=70)$.

Findings: Compared with normal mucosa, LINE-1 in ACF were hypermethylated across all groups $(P<0.0001)$. Furthermore, LINE-1 methylation status in normal colorectal mucosa was independent of the presence of adenoma or CC $(P=0.1072)$, and did not differ depending on the distance to the adenoma or CC. Interestingly, when we compared the LINE-1 methylation status in normal mucosa from different segments of the colorectum, we found higher hypomethylation in the rectum compared with the descending colon $(P<0.0001)$.
\end{abstract}

Conclusions: Overall, our results suggest that global hypomethylation is not present in rectal ACF and argues against the existence of LINE-1 methylation field defect in sporadic colon cancer.

Keywords: Colorectal cancer, Aberrant crypt foci, LINE-1, Methylation, Prevention

\section{Findings}

\section{Background}

Aberrant crypt foci (ACF) are considered the first identifiable preneoplastic lesion in CRC [1]. ACF show features that support their potential role as premalignant lesions, such as dysplasia, monoclonality, and mutations, which are present in adenomas and carcinomas but not in normal colon mucosa [2,3]. High-magnification chromoscopic colonoscopy can detect ACF in vivo [4], and several studies using chromoendoscopy have shown a higher rate of

\footnotetext{
* Correspondence: mpellise@clinic.ub.es; fprunes@clinic.ub.es 'Department of Gastroenterology, Hospital Clínic, Centro de Investigación Biomédica en Red en Enfermedades Hepáticas y Digestivas (CIBEREHD)، IDIBAPS, Barcelona, Catalonia, Spain

Full list of author information is available at the end of the article
}

ACF occurrence in patients with CRC and adenomas compared with healthy individuals [4]. Accordingly, ACF (especially those located in the rectum) have been proposed as a potential biomarker for CRC risk [5]. Histologically, ACF can be classified as hyperplastic or dysplastic [6]. Data from single-center studies have suggested that dysplastic ACF may correlate with CRC risk, as there is an increased frequency of dysplastic ACF in individuals with a normal colon, patients with adenoma, and patients with CRC [1]. However, other studies have shown conflicting findings regarding the clinical value of dysplastic ACF [7-9], and thus, the role of ACF in colorectal carcinogenesis is still under debate. Although rectal ACF have

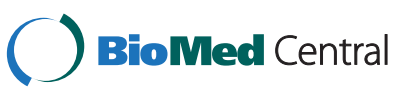

(c) 2014 Quintanilla et al.; licensee BioMed Central Ltd. This is an Open Access article distributed under the terms of the Creative Commons Attribution License (http://creativecommons.org/licenses/by/4.0), which permits unrestricted use, distribution, and reproduction in any medium, provided the original work is properly credited. The Creative Commons Public Domain Dedication waiver (http://creativecommons.org/publicdomain/zero/1.0/) applies to the data made available in this article, unless otherwise stated. 
been suggested as potential biomarkers to identify individuals at high risk for CRC because of their specific histological and molecular features, this is still an area of uncertainty [10].

Methylation of cytosine residues at $\mathrm{CpG}$ dinucleotides is a major epigenetic modification strongly associated with transcriptional silencing. Long interspersed element-1 (LINE-1) elements are a type of genomic repetitive elements that in their full-length form encode the retrotransposon machinery [11]. LINE-1 methylation status is a well-known surrogate marker for genome-wide DNA methylation level. In normal cells, LINE-1 is heavily methylated [12], whereas cancer cells show LINE-1 hypomethylation, leading to chromosomal instability [13]. This phenomenon occurs early in the colorectal carcinogenesis $[14,15]$. However, despite the gradual increase of hypomethylation in the adenoma-carcinoma sequence [16], LINE-1 methylation in ACF has never been studied. Moreover, recent studies have reported a field defect in LINE-1 hypomethylation $[14,17,18]$, suggesting that LINE-1 methylation status in normal mucosa could be used to stratify CRC risk and tailor preventive strategies.

The aims of this study were to analyze the levels of LINE-1 methylation in rectal ACF compared with paired normal colorectal mucosa, and to investigate the putative field defect of LINE-1 hypomethylation by analyzing the normal mucosa of patients from three different CRC risk groups. The analysis was confined to rectal ACF because of their previously reported higher frequency compared with other colonic segments, in addition to their usefulness as CRC biomarkers based on the total number and histological characteristics [14].

\section{Subjects and methods \\ Subjects}

A random selection of patients referred to our hospital for a diagnostic colonoscopy between 2008 and 2010 was prospectively included in this study. Colonoscopy was performed with high-definition endoscopes (H180; Olympus, Tokio, Japan). Based on the colonoscopy findings, individuals were classified into healthy control $(n=70)$, adenoma $(n=42)$, and colon cancer $(C C)(n=35)$ groups. The distal rectum $(10 \mathrm{~cm})$ was explored with narrow band imaging and then with high-resolution chromoendoscopy using methylene blue $0.5 \%$. For each individual, biopsy samples of normal descending colon and rectal mucosa (with a minimum distance of $10 \mathrm{~cm}$ away from any lesion), and up to three ACF in the rectum were used for molecular analyses (Figure 1). Patients with rectal cancer were excluded from the analysis in order to ensure that

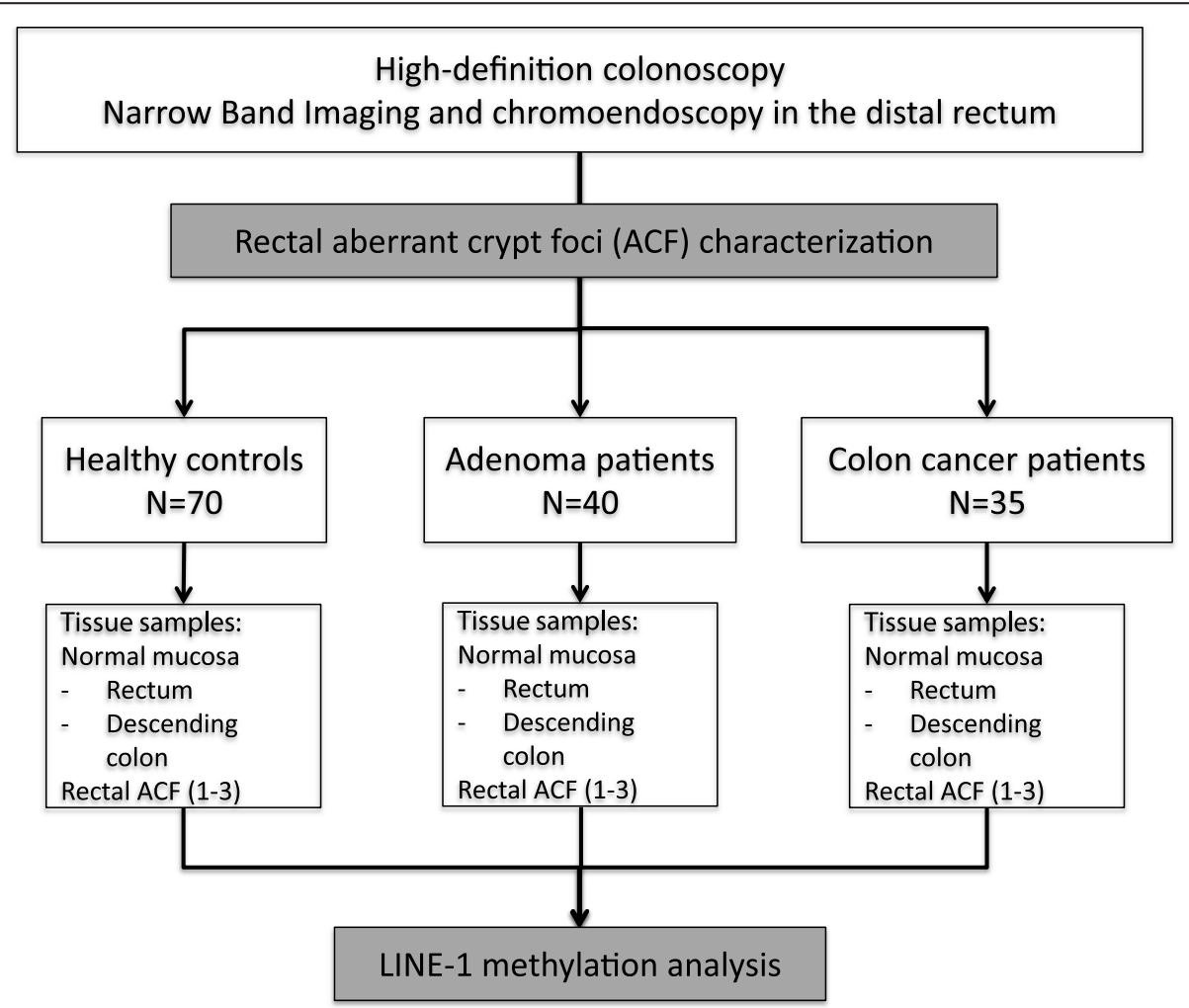

Figure 1 Study design. Flow chart summarizing the study design. Long interspersed element-1 (LINE-1) methylation analysis was performed in DNA extracted from aberrant crypt foci (ACF) and normal colorectal mucosa samples from three colon cancer risk groups (individuals with a normal colonoscopy, patients with adenomas, and patients with colon cancer). 
biopsies were taken at least $10 \mathrm{~cm}$ away from the colorectal neoplasia in all patients. Two different endoscopists registered the number and features of ACF, and two expert pathologists characterized all biopsies.

The project was granted approval from institutional review board of the Hospital Clinic of Barcelona, and written informed consent was obtained from all participants.

\section{DNA extraction}

$\mathrm{ACF}$ and normal mucosa biopsies were preserved at $-80^{\circ} \mathrm{C}$ optimal cutting compound (OCT) and phosphate-buffered saline (PBS), respectively. DNA was extracted using an All Prep DNA/RNA Mini Kit (catalog number 80204; Qiagen, Hilden, Germany) following the manufacturer's recommendations. DNA and RNA were quantified using a NanoDrop Spectrophotometer ND-1000 (Thermo Fisher Scientific).

Bisulfite pyrosequencing and LINE-1 methylation analysis Bisulfite treatment was performed using the EpiTect Bisulfite kit (catalog number 59104; Qiagen) following manufacturer's instructions. Methylation analysis of LINE1 promoter was investigated using pyrosequencing-based methylation analysis [15]. PCR was carried out in a $25 \mu \mathrm{l}$ PCR mixture containing $12.5 \mu \mathrm{l}$ of GoTaq Colorless Master Mix (catalog number M7142, Promega, WI, USA) (Reaction Buffer $\mathrm{pH} 8.5,400 \mu \mathrm{M}$ of each dNTP, and $3 \mathrm{mM} \mathrm{MgC}_{12}$ ), $1 \mu \mathrm{l}$ of Taq polymerase, $2 \mu \mathrm{l}$ of forward primer (5'-TTTTGAGTTAGGTGTGGGATATA-3'), $2 \mu \mathrm{l}$ of reverse biotinylated primer (5'-AAAATCAAAAAA TTCCCTTTC-3'), and $100 \mathrm{ng}$ of bisulfite-treated genomic DNA. PCR cycling conditions were $95^{\circ} \mathrm{C}$ for $15 \mathrm{mi}-$ nutes; 45 cycles of $94^{\circ} \mathrm{C}$ for 30 seconds, $55^{\circ} \mathrm{C}$ for 45 seconds, and $72^{\circ} \mathrm{C}$ for 45 seconds; and finally, $10 \mathrm{mi}-$ nutes at $72^{\circ} \mathrm{C}$ and $4^{\circ} \mathrm{C}$ forever. The biotinylated PCR product was purified and made single-stranded to act as a template in a pyrosequencing reaction as recommended by the manufacturer using Pyrosequencing Vacuum Tool (Qiagen, Hilden, Germany). In brief, the PCR product was bound to a Streptavidin Sepharose HP column, and the sepharose beads containing the immobilized PCR product were purified, washed, denatured using $0.2 \mathrm{M} \mathrm{NaOH}$ solution, and washed again. Then, $10 \mu \mathrm{M}$ of pyrosequencing primer (5'AGTTAGGTGTGGGATATAGT-3') was annealed to the purified single-stranded PCR product, and pyrosequencing was performed using a PSQ 96MA Pyrosequencing System (Qiagen). CpGenome Methylated DNA (catalog number S7821; Millipore, Darmstadt, Germany) was used as a positive control. Methylation level of LINE-1 elements was calculated as the mean percentage of the four $\mathrm{CpG}$ sites analyzed, as previously described [19].

\section{Statistical analysis}

Distribution of LINE-1 methylation levels was assessed with the Shapiro-Wilks test, which showed that the data were not normally distributed $(P=0.017)$. Accordingly, the Wilcoxon signed-rank test was used for comparing average methylation levels of paired ACF and normal mucosa. When comparing global methylation between two independent groups and between more than two groups, the Mann-Whitney $U$-test and Kruskal-Wallis test, respectively, were used. LINE-1 methylation levels are expressed as median and interquartile range (IQR). All statistical analyses were performed using SPSS software (v20; IBM Inc., Armonk, NY, USA) and results were considered significant at $P<0.05$.

\section{Results}

Baseline characteristics of patients and rectal ACF related to $\mathrm{CRC}$ risk group are detailed in Table 1 . As shown, the adenoma group mainly comprised patients with advanced adenomas (mean size $12.3 \pm 9.8 \mathrm{~mm}$ ) located throughout the colorectum. Although 16 patients (40\%) with adenoma had synchronous serrated polyps, the vast majority of them were small hyperplastic polyps located in the rectosigmoid colon, which are known to lack clinical significance.

\section{Rectal ACF do not display LINE-1 hypomethylation}

To test the role of rectal ACF as a potential precursor lesion, we analyzed the LINE-1 methylation levels in rectal ACF and paired normal rectal and descending colon mucosa from all patients. The median LINE-1 methylation level of up to three ACF for each patient was considered for statistical purposes using the Wilcoxon signed-rank test. As shown in Figure 2A, our data indicated that overall, levels of LINE-1 methylation were higher in ACF than in normal rectal mucosa $(80.02 \%$ (77.54 to 82.22$)$ versus $76.13 \%$ (73.74 to 78.06 ); $P<0.0001$ ). In fact, only 20 subjects exhibited lower LINE-1 methylation in their rectal ACF compared with their normal mucosa, and LINE-1 in rectal ACF was not hypomethylated in any of the three risk groups (Figure 2B). Likewise, similar results were observed for LINE-1 methylation levels when comparing rectal ACF and normal mucosa from descending colon $[80.02 \%$ (77.54 to 82.22 ) versus $78.42 \%$ (76.51 to 80.33 ]; $P=0.0002$ ) (see Additional file 1: Figure S1). We also performed these analyses stratifying by sex, with similar results for both males and females (see Additional file 2: Table S1). Interestingly, there were significant differences in LINE-1 methylation between dysplastic ACF and hyperplastic ACF [81.46\% (78.75 to 82.97 ) versus $79.54 \%$ (76.88 to 81.79 ); $P=0.031$ ].

\section{LINE-1 methylation levels in normal colorectal mucosa are} similar in all CC risk groups

To assess the LINE-1 field defect, we compared the methylation status of LINE-1 in the normal rectal and 
Table 1 Baseline characteristics of individuals and aberrant crypt foci in each colon cancer risk group

\begin{tabular}{|c|c|c|c|}
\hline & Control $(n=70)$ & Adenoma $(n=42)$ & Colon cancer $(n=35)$ \\
\hline \multicolumn{4}{|l|}{ Sex, n (\%) } \\
\hline - Female & $48(68.6 \%)$ & $17(40.5 \%)$ & $13(37.1 \%)$ \\
\hline - Male & $22(31.4 \%)$ & $25(59.5 \%)$ & $22(62.9 \%)$ \\
\hline Age, years, mean $\pm S D$ & $58.1 \pm 15.3$ & $68.6 \pm 9.9$ & $66.8 \pm 8.6$ \\
\hline \multicolumn{4}{|l|}{ Colon cancer location, n (\%) } \\
\hline - Sigmoid & - & - & $21(60 \%)$ \\
\hline - Descending & - & - & $2(5.7 \%)$ \\
\hline - Transverse & - & - & $1(2.9 \%)$ \\
\hline - Ascending & - & - & $9(25.7 \%)$ \\
\hline - Cecum & - & - & $2(5.7 \%)$ \\
\hline \multicolumn{4}{|l|}{ Colon cancer TNM stage, n (\%) } \\
\hline-1 & - & - & $10(28.6 \%)$ \\
\hline$-\|$ & - & - & $11(31.4 \%)$ \\
\hline$-|I|$ & - & - & $13(37.1 \%)$ \\
\hline$-I V$ & - & - & $1(2.9 \%)$ \\
\hline \multicolumn{4}{|l|}{ Adenoma features (per patient) ${ }^{a}$} \\
\hline - Tubular, n (\%) & - & $28(70 \%)$ & $11(31.4 \%)$ \\
\hline - Tubulovillous, n (\%) & - & $7(17.5 \%)$ & $1(2.9 \%)$ \\
\hline - Villous, n (\%) & - & $5(12.5 \%)$ & $0(0 \%)$ \\
\hline - High-grade dysplasia, n (\%) & - & $11(27.5 \%)$ & $5(14.3 \%)$ \\
\hline - Adenoma size, mean $(\mathrm{mm}) \pm S D$ & - & $12.3 \pm 9.8$ & $8.4 \pm 5$ \\
\hline - Number of adenomas per patient, mean \pm SD (range) & - & $2.4 \pm 2(1$ to 9$)$ & $1.2 \pm 1.2(1$ to 4$)$ \\
\hline Advanced adenoma ${ }^{\mathrm{b}}, \mathrm{n}(\%)$ & & $22(55 \%)$ & $7(20 \%)$ \\
\hline \multicolumn{4}{|l|}{ Adenoma location (per patient) ${ }^{c}, \mathrm{n}(\%)$} \\
\hline - Rectum & - & $12(30 \%)$ & $2(5.7 \%)$ \\
\hline - Sigmoid & - & $10(25 \%)$ & $3(8.6 \%)$ \\
\hline - Descending & - & $5(12.5 \%)$ & $3(8.6 \%)$ \\
\hline - Transverse & - & $5(12.5 \%)$ & $2(5.7 \%)$ \\
\hline - Ascending & - & $5(12.5 \%)$ & $2(5.7 \%)$ \\
\hline - Cecum & - & $3(7.5 \%)$ & $0(0 \%)$ \\
\hline \multicolumn{4}{|l|}{ Serrated polyps features (per patient) ${ }^{d}$} \\
\hline - Recto-sigmoid hyperplastic polyps, n (\%) & - & $12(30 \%)$ & $3(8.6 \%)$ \\
\hline - Proximal serrated polyps, n (\%) & - & $4(10 \%)$ & $5(14.3 \%)$ \\
\hline - Serrated polyps $\geq 10$ mm, n (\%) & - & $2(5 \%)$ & $2(5.7 \%)$ \\
\hline - Serrated polyps per patient, $n$, mean \pm SD (range) & - & $1.6 \pm 0.8$ (1 to 3$)$ & $3.1 \pm 3.4(1$ to 5$)$ \\
\hline - Hyperplastic polyp, n (\%) & - & $15(37.5 \%)$ & $8(22.9 \%)$ \\
\hline - Sessile serrated adenoma, n (\%) & - & $1(\%)$ & $0(0 \%)$ \\
\hline -Traditional serrated adenoma, n (\%) & - & $0(\%)$ & $0(0 \%)$ \\
\hline
\end{tabular}


Table 1 Baseline characteristics of individuals and aberrant crypt foci in each colon cancer risk group (Continued)

\begin{tabular}{lccc}
\hline ACF for molecular study, $\mathrm{n}$ & 117 & 76 & 77 \\
Dysplastic ACF, $\mathrm{n}(\%)$ & $24(17.4 \%)$ & $23(24 \%)$ & $17(25 \%)$ \\
Hyperplastic ACF, $\mathrm{n}(\%)$ & $114(82.6 \%)$ & $73(76 \%)$ & $51(75 \%)$ \\
\hline
\end{tabular}

$\mathrm{ACF}$, aberrant crypt foci; SD, standard deviation.

${ }^{a}$ This referred to 40 individuals in the adenoma group for whom full pathological information about the adenomas was available.

${ }^{\mathrm{b}}$ Advanced adenoma: $\geq 10 \mathrm{~mm}$ in size, or presence of high-grade dysplasia or villous features.

'In cases with $>1$ adenoma, this referred to the location of the most advanced adenoma.

dincluded hyperplastic polyps, sessile serrated adenomas, and traditional serrated adenomas.

eproximal to the sigmoid colon.

descending colon mucosa of individuals from the three CC risk groups (CC, adenoma and healthy controls). Because the healthy control group was significantly younger than the adenoma and CC groups (Table 1), we first excluded an age effect in the level of LINE-1 methylation in normal mucosa (see Additional file 3: Figure S2). As shown in Figure 3A, methylation of LINE-1 in normal rectal mucosa

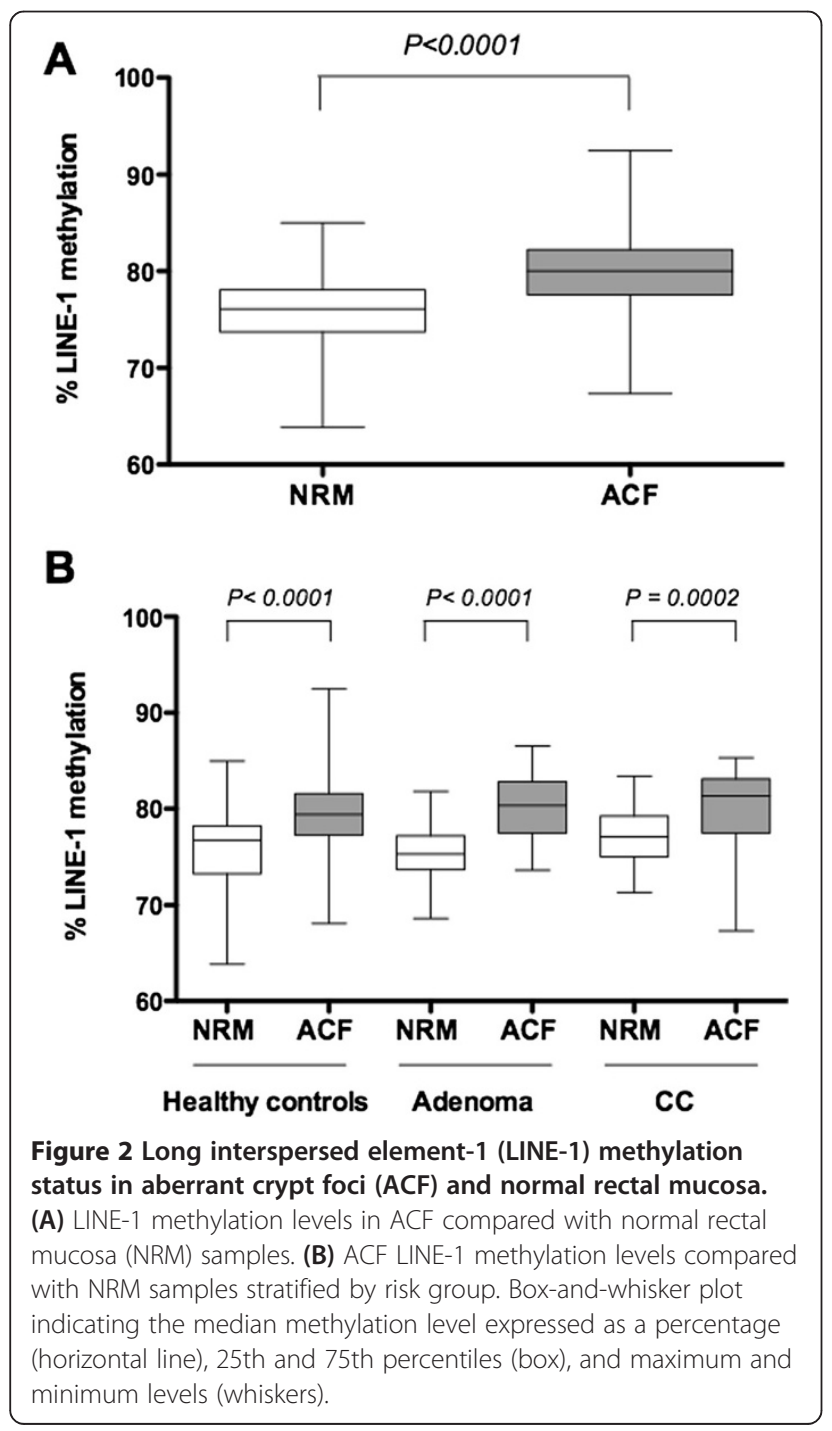

was independent of the patient group [healthy controls: 76.73\% (73.23 to 78.22 ); adenoma: $75.34 \%$ (73.7 to 77.2 ); CC: $77.06 \%$ (75.04 to 79.23$) ; P=0.107$ ] (Table 2). Comparable results were obtained when normal mucosa from the descending colon was considered [healthy controls: $77.67 \%$ (75.84 to 80.18 ); adenoma: $78.45 \%$ (77.37 to 79.92$)$; CC: $80.30 \%$ (77.19 to 81.39 ); $P=0.147$ ] (Table 2). These analyses were also performed with stratification by sex, with similar results (see Additional file 2: Table S1).

We also analyzed the potential field defect for LINE-1 methylation by stratifying the results by the distance of the lesion to the normal mucosa biopsies. As shown in Table 3, LINE-1 methylation levels in normal mucosa

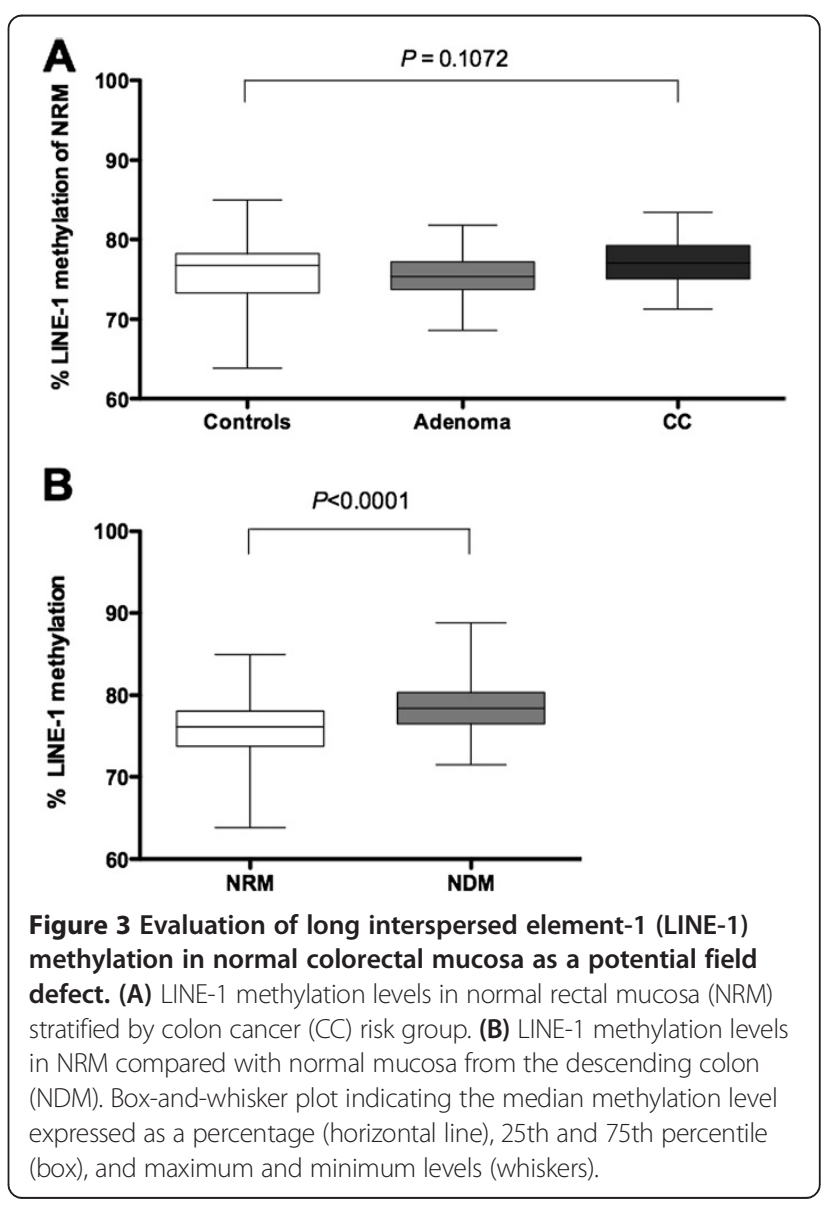


Table 2 LINE-1 methylation levels in normal mucosa and aberrant crypt foci for each of the three groups of subjects

\begin{tabular}{|c|c|c|c|c|}
\hline & Healthy controls $(n=70)$ & Adenoma $(n=42)$ & Colon cancer $(n=35)$ & $P$ value $^{\mathrm{a}}$ \\
\hline Normal rectal mucosa, n/N & $62 / 70$ & $31 / 42$ & $24 / 35$ & 0.107 \\
\hline Median (IQR) & $76.73 \%$ (73.23 to 78.22$)$ & $75.34 \%$ (73.7 to 77.2$)$ & $77.06 \%$ (75.04 to 79.23$)$ & \\
\hline Normal descending colon mucosa, n/N & $42 / 70$ & $38 / 42$ & $21 / 35$ & 0.147 \\
\hline Median (IQR) & $77.67 \%$ (75.84 to 80.18$)$ & $78.45 \%$ (77.37 to 79.92$)$ & $80.30 \%$ (77.19 to 81.39$)$ & \\
\hline Aberrant crypt foci, n/N & $68 / 70$ & $38 / 42$ & $24 / 35$ & 0.409 \\
\hline Median (IQR) & $79.41 \%$ (77.24 to 81.58$)$ & $80.32 \%$ (77.45 to 82.82$)$ & $81.37 \%$ (77.48 to 83.14$)$ & \\
\hline Dysplastic aberrant crypt foci, n/N & 15 & 11 & 9 & 0.32 \\
\hline Median (IQR) & $80.58 \%$ (78.54 to 82.37$)$ & $80.99 \%$ (79.15 to 83.07$)$ & $82.74 \%$ (79.83 to 83.43$)$ & \\
\hline Hyperplastic aberrant crypt foci, n/N & 53 & 27 & 24 & 0.812 \\
\hline Median (IQR) & $79.32 \%$ (76.94 to 81.54$)$ & $79.59 \%$ (76.80 to 82.22$)$ & $80.02 \%$ (76.50 to 82.55$)$ & \\
\hline
\end{tabular}

IQR, interquartile range; $n$, number of samples analyzed in each group; $N$, total number of samples included in each group.

${ }^{a}$ Kruskal-Wallis test was used to assess the level of LINE-1 methylation between the three risk groups.

did not differ depending on the distance to the adenoma or CC.

\section{LINE-1 methylation levels in normal mucosa differ between colonic segments}

Interestingly, when we compared the LINE-1 methylation status in normal mucosa from different segments of the colorectum, we found a higher degree of hypomethylation in the rectum compared with the descending colon [76.13\% (73.75 to 78.06 ) versus $78.42 \%$ (76.51 to 80.33); $P<0.0001$ ], suggesting different genome-wide methylation profiles between normal colon and rectal mucosa (Figure 3B). Stratification by sex did not vary the results (see Additional file 2: Table S1).

\section{Discussion}

Overall, our results argue against the hypothesis that global hypomethylation occurs at the earliest stages of colorectal carcinogenesis. Overexpression of $\beta$-catenin and KRAS mutations may indicate that ACF constitute a premalignant stage [16]. Moreover, LINE-1 hypomethylation is already present at the adenoma stage [15]. Our data suggest, for the first time, to our knowledge, that the formation of ACF is not triggered by hypomethylation in LINE-1 elements, and that genome-wide hypomethylation occurs after ACF formation, most likely during the transition from non-adenomatous to adenomatous epithelia. In fact, hypermethylation of LINE-1 elements in ACF suggests that reversal epigenetic changes occur in these lesions regardless of their pathogenic progression.

Despite the fact that two previous reports have suggested the existence of LINE-1 hypomethylation field defects in CRC, our results indicate the absence of this phenomenon in our sample set. Kamiyama and collaborators recently showed that normal colonic mucosa from patients with synchronous CRC displayed higher hypomethylation of LINE-1 compared with patients with solitary $\mathrm{CRC}$ and controls [17]. However, and in line with our results, there were no differences between the two latter groups. Another study has shown that normal colorectal mucosa samples from different risk groups displayed differences in LINE-1 methylation that mirrored differences between their respective tumor specimens [17]. A more recent study has shown that adjacent mucosa from individuals with multiple/large serrated polyps display significantly lower LINE-1 methylation levels compared with individuals without such polyps [18]. We tested the hypothesis that LINE-1 methylation in normal mucosa could be used as a biomarker for risk of sporadic CRC, focusing on patients with conventional adenomas. By analyzing a prospective cohort of patients with $\mathrm{CC}$ and controls, we found that our data suggest that methylation status of LINE-1 in normal mucosa is not a suitable biomarker to predict CC risk in patients with sporadic cancer. However, the potential field defect for LINE-1 methylation in patients with serrated polyps needs further assessment.

Table 3 LINE-1 methylation levels in normal mucosa stratifying by location of the neoplastic lesion

\begin{tabular}{|c|c|c|c|c|c|c|}
\hline LINE-1 methylation & $\begin{array}{l}\text { Patients with proximal } \\
\text { adenoma }(\mathrm{n}=10)^{\mathrm{a}}\end{array}$ & $\begin{array}{l}\text { Patients with distal } \\
\text { adenoma }(\mathrm{n}=30)^{\mathrm{a}}\end{array}$ & $P$ value & $\begin{array}{l}\text { Patients with proximal } \\
\text { colon cancer }(n=11)^{a}\end{array}$ & $\begin{array}{l}\text { Patients with distal } \\
\text { colon cancer }(n=24)^{a}\end{array}$ & $P$ value \\
\hline Normal rectal mucosa & $73.87 \%$ (72.47 to 75.90$)$ & $76.06 \%$ (74.78 to 77.33$)$ & 0.087 & $77.59 \%$ (72.78 to 80.12$)$ & $77.40 \%$ (75.70 to 79.79$)$ & 0.794 \\
\hline $\begin{array}{l}\text { Normal descending } \\
\text { mucosa }\end{array}$ & $80.72 \%$ (76.62 to 82.68) & $78.38 \%$ (76.55 to 80.01$)$ & 0.176 & $77.14 \%$ (75.59 to 80.06$)$ & $78.59 \%$ (76.90 to 79.41$)$ & 0.458 \\
\hline
\end{tabular}

LINE-1 methylation levels are expressed as median (interquartile range, IQR).

${ }^{\text {a }}$ Referred to the splenic flexure. 
In addition, our data show different methylation levels in normal mucosa between the descending colon and the rectum, suggesting different methylation profiling depending on the colonic segment.

\section{Conclusion}

Our results shed some light on the role of genome-wide methylation in rectal ACF and argue against its utility as a biomarkers for assessing $\mathrm{CC}$ risk.

\section{Additional files}

Additional file 1: Figure S1. LINE-1 methylation status in aberrant crypt foci and normal mucosa from the descending colon. (A) LINE-1 methylation levels in aberrant crypt foci (ACF) compared to normal mucosa from the descending colon (NDM). (B) ACF LINE-1 methylation levels compared to descending mucosa samples according to risk group. Box-and-whisker plot indicating the median methylation level expressed as a percentage (horizontal line), $25^{\text {th }}$ and $75^{\text {th }}$ percentile (box), and maximum and minimum levels (whiskers).

Additional file 2: Table S1. LINE-1 methylation analysis stratified by sex Additional file 3: Figure S2. Evaluation of the age-effect for LINE-1 methylation data. Representation of the correlation between LINE-1 methylation levels in normal rectal mucosa and the age of patients.

\section{Abbreviations}

ACF: Aberrant crypt foci; CC: Colon cancer; CRC: Colorectal cancer; LINE-1: Long interspersed element-1; NDM: Normal descending mucosa; NRM: Normal rectal mucosa.

\section{Competing interests}

The authors declare that they have no competing interests.

\section{Authors' contributions}

$M P, M L-C, I Q$ and FB participated in the design and coordination of the study. MP, AC, and FB supervised the study. MJ and MC carried out the pathological analysis of the samples. ML-C, LM, SC, and MLL participated in the acquisition of data. IQ, MP, and FB carried out the molecular genetic studies and their interpretation, and drafted the manuscript. AC, JC, MP, and FB revised the manuscript. All authors read and approved the final manuscript.

\section{Authors' information}

$M P$ and FB share senior authorship.

\section{Acknowledgments}

This work was supported by a grant from the Fondo de Investigaciones Sanitarias (FIS) (Proyectos de Evaluación de Tecnologías Sanitarias PS09/00669); the Banc de Tumors-Biobanc Hospital Clinic-IDIBAPS and Xarxa de Bancs de Tumors de Catalunya (XBTC). The Centro de Investigación Biomédica en Red de Enfermedades Hepáticas y Digestivas (CIBEREHD) is funded by Instituto de Salud Carlos III. ML-C was a Research Fellow from the FIS (Rio Hortega contract). IQ is funded by the Ministerio de Educación (FPU2012 program). FB is funded by Instituto de Salud Carlos III (PI10/00384 and PI13/00719). MP is funded by Instituto de Salud Carlos III (PI12/01481); "Cofinanciado por el Fondo Europeo de Desarrollo Regional (FEDER). Unión Europea. Una manera de hacer Europa". JC was awarded a grant from the Asociación Española Contra el Cáncer, and is supported by Instituto de Salud Carlos III (CP13/00160).

\section{Author details}

${ }^{1}$ Department of Gastroenterology, Hospital Clínic, Centro de Investigación Biomédica en Red en Enfermedades Hepáticas y Digestivas (CIBEREHD), IDIBAPS, Barcelona, Catalonia, Spain. ${ }^{2}$ Pathology Department, Centre de Diagnostic Biomèdic (CDB), Hospital Clínic, University of Barcelona and Banc de Tumors-Biobanc Clinic-IDIBAPS-XBTC, Barcelona, Catalonia, Spain.
Received: 14 August 2014 Accepted: 17 October 2014

Published: 10 November 2014

\section{References}

1. Takayama T, Katsuki S, Takahashi Y, Ohi M, Nojiri S, Sakamaki S, Kato J, Kogawa K, Miyake H, Niitsu Y: Aberrant crypt foci of the colon as precursors of adenoma and cancer. N Engl J Med 1998, 339:1277-1284

2. McLellan EA, Medline A, Bird RP: Dose response and proliferative characteristics of aberrant crypt foci: putative preneoplastic lesions in rat colon. Carcinogenesis 1991, 12:2093-2098.

3. Bird RP, Good CK: The significance of aberrant crypt foci in understanding the pathogenesis of colon cancer. Toxicol Lett 2000, 112-113:395-402.

4. Yokota T, Sugano K, Kondo H, Saito D, Sugihara K, Fukayama N, Ohkura H, Ochiai A, Yoshida S: Detection of aberrant crypt foci by magnifying colonoscopy. Gastrointest Endosc 1997, 46:61-65.

5. Gupta S, Ashfaq R, Kapur P, Afonso BB, Nguyen TP, Ansari F, Boland CR, Goel A, Rockey DC: Microsatellite instability among individuals of Hispanic origin with colorectal cancer. Cancer 2011, 116:4965-4972.

6. Carr NJ SL, Neiderau C: Aberrrant crypt foci. Pathology and genetics: Tumors of the digestive system. In WHO Classification of Tumours Series. Lyon: IARC Press; 2000.

7. Adler DG, Gostout CJ, Sorbi D, Burgart LJ, Wang L, Harmsen WS: Endoscopic identification and quantification of aberrant crypt foci in the human colon. Gastrointest Endosc 2002, 56:657-662.

8. Pretlow TP, Pretlow TG: Mutant KRAS in aberrant crypt foci (ACF): initiation of colorectal cancer? Biochim Biophys Acta 2005, 1756:83-96.

9. Suehiro Y, Hinoda Y: Genetic and epigenetic changes in aberrant crypt foci and serrated polyps. Cancer Sci 2008, 99:1071-1076.

10. Mutch MG, Schoen RE, Fleshman JW, Rall CJ, Dry S, Seligson D, Charabaty A, Chia D, Umar A, Viner J, Hawk E, Pinsky PF: A multicenter study of prevalence and risk factors for aberrant crypt foci. Clin Gastroenterol Hepatol 2009, 7:568-574

11. Speek M: Antisense promoter of human L1 retrotransposon drives transcription of adjacent cellular genes. Mol Cell Biol 2001, 21:1973-1985.

12. Kazazian HH Jr: Mobile elements: drivers of genome evolution. Science 2004, 303:1626-1632.

13. Howard G, Eiges R, Gaudet F, Jaenisch R, Eden A: Activation and transposition of endogenous retroviral elements in hypomethylation induced tumors in mice. Oncogene 2008, 27:404-408.

14. Pavicic W, Joensuu El, Nieminen T, Peltomaki P: LINE-1 hypomethylation in familial and sporadic cancer. J Mol Med (Berl) 2012, 90:827-835.

15. Sunami E, de Maat M, Vu A, Turner RR, Hoon DS: LINE-1 hypomethylation during primary colon cancer progression. PLOS One 2011, 6:e18884.

16. Ibrahim AE, Arends MJ, Silva AL, Wyllie AH, Greger L, Ito Y, Vowler SL, Huang $\mathrm{TH}$, Tavare S, Murrell A, Brenton JD: Sequential DNA methylation changes are associated with DNMT3B overexpression in colorectal neoplastic progression. Gut 2011, 60:499-508.

17. Kamiyama H, Suzuki K, Maeda T, Koizumi K, Miyaki Y, Okada S, Kawamura YJ, Samuelsson JK, Alonso S, Konishi F, Perucho M: DNA demethylation in normal colon tissue predicts predisposition to multiple cancers. Oncogene 2012, 31:5029-5037.

18. Yamada A, Minamiguchi S, Sakai Y, Horimatsu T, Muto M, Chiba T, Boland CR, Goel A: Colorectal advanced neoplasms occur through dual carcinogenesis pathways in individuals with coexisting serrated polyps. PLoS One 2014, 9:e98059.

19. Antelo M, Balaguer F, Shia J, Shen Y, Hur K, Moreira L, Cuatrecasas M, Bujanda L, Giraldez MD, Takahashi M, Cabanne A, Barugel ME, Arnold M, Roca EL, Andreu M, Castellvi-Bel S, Llor X, Jover R, Castells A, Boland CR, Goel A: A high degree of LINE-1 hypomethylation is a unique feature of early-onset colorectal cancer. PLoS One 2012, 7:e45357.

doi:10.1186/1868-7083-6-24

Cite this article as: Quintanilla et al:: LINE-1 hypomethylation is neither present in rectal aberrant crypt foci nor associated with field defect in sporadic colorectal neoplasia. Clinical Epigenetics 2014 6:24. 